PHYSICAL REVIEW D 94, 065004 (2016)

\title{
Leptogenesis with high-scale electroweak symmetry breaking and an extended Higgs sector
}

\author{
Laura Covi \\ Institut für Theoretische Physik, Friedrich-Hund-Platz 1, D-37077 Göttingen, Germany and CERN, \\ CH 1211 Geneva 23, Switzerland \\ Jihn E. Kim \\ Department of Physics, Kyung Hee University, 26 Gyungheedaero, Dongdaemun-Gu, Seoul 02447, \\ Republic of Korea, and Center for Axion and Precision Physics Research (IBS), \\ 291 Daehakro, Yuseong-Gu, Daejeon 34141, Republic of Korea \\ Bumseok Kyae \\ Department of Physics, Pusan National University, 2 Busandaehakro-63-Gil, Geumjeong-Gu, Busan \\ 46241, Republic of Korea and School of Physics, Korea Institute for Advanced Study, 85 Hoegiro, \\ Dongdaemun-Gu, Seoul 02455, Republic of Korea \\ Soonkeon Nam \\ Department of Physics, Kyung Hee University, 26 Gyungheedaero, Dongdaemun-Gu, \\ Seoul 02447, Republic of Korea \\ (Received 26 April 2016; published 7 September 2016)
}

\begin{abstract}
We propose a new scenario for baryogenesis through leptogenesis, where the $C P$ phase relevant for leptogenesis is connected directly to the Pontecorvo-Maki-Nakagawa-Sakada (PMNS) phase(s) in the light neutrino mixing matrix. The scenario is realized in the case when only one $C P$ phase appears in the full theory, originating from the complex vacuum expextation value of a standard model singlet field. In order to realize this scheme, the electroweak symmetry is required to be broken during the leptogenesis era, and a new loop diagram with an intermediate $W$ boson exchange including the low-energy neutrino mixing matrix should play the dominant contribution to the $C P$ violation for leptogenesis. In this article, we discuss the new basic mechanism, which we call type-II leptogenesis, and give an estimate for maximally reachable baryon asymmetry depending on the PMNS phases.
\end{abstract}

DOI: $10.1103 /$ PhysRevD.94.065004

\section{INTRODUCTION}

The origin of the baryon asymmetry of the Universe (BAU) has been a longstanding theoretical issue [1]. Among Sakharov's three conditions [2] for successful generation of an asymmetry from a symmetric initial state, the first, i.e., baryon number violation, and the second, i.e., $\mathrm{C}$ and $C P$ violation, rely most strongly on model building beyond the standard model (SM) of particle physics. Along this line, there already exist plenty of theoretical models to generate the BAU [3-7], with different ways to depart from thermal equilibrium, e.g., from heavy particle decay outside equilibrium to first-order phase transitions or to the dynamical Affleck-Dine (AD) mechanism [5].

In this article, we would like to follow an alternative route, realizing instead the leptogenesis mechanism within

Published by the American Physical Society under the terms of the Creative Commons Attribution 3.0 License. Further distribution of this work must maintain attribution to the author(s) and the published article's title, journal citation, and DOI. a phase with broken electroweak symmetry at high temperature and relying mostly on SM physics in the neutrino sector to achieve the necessary $C P$ violation. The only ingredients beyond the SM that we need is the presence of various species of SM singlets, with the quantum numbers of right-handed $(\mathrm{RH})$ neutrinos, different Higgs doublets, in order to allow for a nonvanishing contribution to the $C P$ asymmetry from a $W$-boson loop and to keep the electroweak symmetry broken.

Indeed, at the level of the SM of particle physics, $C P$ violation is related to the charged-current interaction and determined by two $C P$ phases-one in the quark sector, the Cabibbo-Kobayashi-Maskawa $(\mathrm{CKM})$ phase $\delta_{\mathrm{CKM}}[8,9]$, and the other, the Pontecorvo-Maki-Nakagawa-Sakada (PMNS) phase $\delta_{\text {PMNS }}[10]$, while two more Majorana phases are appearing in the leptonic sector. In a family unified grand unified theory (GUT), these two phases can be related if only one single complex vacuum expectation value (VEV) appears in the full theory [11]. From the early time on, it has been an interesting issue to investigate the possibility of relating the baryon asymmetry with the SM phase(s) $\delta_{\mathrm{CKM}}$ and/or $\delta_{\mathrm{PMNS}}$. 
The first obvious possibility is to exploit $\delta_{\mathrm{CKM}}$ for the BAU, but it has been known for a long time that such a phase, appearing always with small mixing angles, is not enough for the baryon number generation in GUT baryogenesis [12]. Even in other scenarios, relying on the quark sector, like the $\mathrm{AD}$ mechanism [5] through the baryon number carrying scalars or the electroweak baryogenesis, additional $C P$ violating phases are needed to provide a large enough baryon asymmetry [13]. Therefore, it is difficult to explain the BAU just considering the $C P$ violation in the quark sector.

A more promising road is given by baryogenesis through leptogenesis [6], since the phases in the leptonic sector are unconstrained and the mixings large. This mechanism relies on the fact that sphaleron processes are effective before and during the electroweak phase transition and violate both baryon $(B)$ and lepton $(L)$ numbers, but conserve baryon minus lepton number $(B-L)$. Therefore, baryogenesis or leptogenesis above the electroweak scale must generate a nonvanishing $B-L$ number that is then translated into a baryon asymmetry before or at the electroweak transition.

In this paper, we consider a leptogenesis scenario, which allows us to relate the $C P$ violation during leptogenesis to the phase $\delta_{\text {PMNS }}$ in the light neutrino mixing matrix. In order to be able to have a well-defined neutrino mixing matrix when the lepton asymmetry is cosmologically created, we require that the $\mathrm{SM}$ gauge group $\mathrm{SU}(2)_{L} \times$ $\mathrm{U}(1)_{Y}$ remains broken during the leptogenesis epoch. In fact, the Brout-Englert-Higgs(BEH) mechanism for $\mathrm{SU}(2)_{L} \times \mathrm{U}(1)_{Y}$ breaking at high temperature is possible for some regions in the parameter space of BEH bosons $h_{u}$ and $h_{d}$ [14]. With the radiative breaking of the SM in supergravity [15], the high temperature breaking of $\mathrm{SU}(2) \times \mathrm{U}(1)$ can be quite generic. This high-temperature effect occurs at a scale somewhat below the SUSY breaking scale of order $10^{11} \mathrm{GeV}$.

\section{A NEW TYPE OF LEPTOGENESIS}

In the leptogenesis scheme with one or two BEH doublets, the lepton asymmetry arises from the decay of the lightest heavy Majorana neutrino $N_{1}$ producing light leptons and antileptons and the Higgs particle by the decay

$$
N_{1} \rightarrow \ell_{i}+h_{u}, \quad \bar{\ell}_{i}+h_{u}^{*},
$$

where $\ell_{i}\left(\bar{l}_{i}\right)$ is the $i$ th lepton (antilepton) doublet and $h_{u}$ is the up-type $Y=1 / 2 \mathrm{BEH}$ doublet. We follow here the supersymmetric notation, but the mechanism can work also without supersymmetry. In this case, therefore, the same mother particle $N$ has two decaying channels with different lepton number, and, therefore, the model satisfies the Nanopoulos-Weinberg theorem
$[16,17]$. In classical leptogenesis, the $C P$ violation in the decay arises from the interference of the tree-level with the one-loop diagrams involving the heavier RH neutrinos $N_{j}, j=2,3$ (for the case of three generations). In general, the $C P$ violation arises from the complex Yukawa couplings and has no direct relation to the low-energy $C P$ phases [18], apart from particular textures [19] or $C P$ conservation in the heavy RH neutrino sector $[19,20]$.

In this article, we would like to extend the model in order to have a large contribution to the $C P$ violation from an electroweak loop involving explicitly the PMNS matrix. In order to do so, we introduce another copy of the Higgs doublet $H_{u}$, heavier than the SM one $h_{u}$, and with vanishing $\mathrm{VEV}$, as well as another generation of $\mathrm{RH}$ neutrinos $\mathcal{N}_{1}$. All these particles can mix with $h_{u}, N_{1}$, respectively, and allow for the presence of the diagram in Fig. 1(b), where the virtual particles are all SM particles and one of the vertices include the PMNS matrix directly. For simplicity, we consider here the case where the field $H_{u}$ is heavier than the right-handed neutrino, so that the decay of $N$ into $\ell_{i}+H_{u}$ is negligible.

The $C P$ phase in the PMNS matrix is required to descend down from the high-energy scale by a complex VEV. To relate different phases, we assume that only one SM singlet field $X$ develops a $C P$ phase $\delta_{\mathrm{X}}$. Thus, all Yukawa couplings and the other VEVs are real and all $C P$ violation parameters arise from $\delta_{\mathrm{X}}$.

While the SM Higgs doublet(s) do not carry lepton number, we define the fields $H_{d}$ and $H_{u}$ to carry the lepton number $L=+2$ and -2 , respectively, and $\mathcal{N}$ instead to have $L=1$, while $N$ will be defined to carry $L=-1$. We can then write for the Higgs doublets and the heavy neutrinos the Yukawa couplings:

$$
f N_{1} h_{u} \ell_{L}, \quad \tilde{f} \mathcal{N}_{1} H_{u} \ell_{L} .
$$

The Yukawa couplings ( $\tilde{f}$ 's) of the inert Higgs doublets $H_{u, d}$ to the lepton doublets are distinguished from those ( $f$ 's) of the BEH doublets $h_{u, d}$, and no mixing is allowed at this level due to the different lepton number assignments. We also have other lepton number conserving interactions such as

$$
\Delta m_{0} N_{1} \mathcal{N}_{1}+\mu_{H}^{2} H_{u} H_{d}+\text { H.c. }
$$

where $\Delta m_{0}$ is real. The first term of (3) gives directly a Majorana mass term between $N_{1}$ and $\mathcal{N}_{1}$ without a phase because we defined it preserving the lepton number. The Dirac mass for the seesaw neutrino mass is via $N_{1} h_{u} \ell_{L}$ which appears as in the type-I leptogenesis. The needed lepton number violating couplings are introduced by the couplings

$$
\Delta \mathcal{L} \ni \mu^{\prime 2} h_{u}^{*} H_{u}+m_{0}^{\prime} N_{1} N_{1}+m_{0}^{\prime \prime} \mathcal{N}_{1} \mathcal{N}_{1}+\text { H.c. },
$$


which also allow for mixing in the Higgs sector and for the see-saw mechanism. There are more $L$-violating terms such as $h_{d} H_{u}, h_{u} H_{d}$, and $h_{d}^{*} H_{d}$, which are not relevant for leptogenesis. We will assume $\Delta m_{0} \gg m_{0}^{\prime}, m_{0}^{\prime \prime}$; i.e., the $L$ conserving mass parameter is much larger than the $L$ violating mass parameters. In this case, $\left(N_{1}, \mathcal{N}_{1}\right)$ are maximally mixed, and we call $N$ the lightest mass eigenstate obtained from the mixing of these two states. We can then define an effective Yukawa coupling for this lightest $\mathrm{RH}$ neutrino as

$f^{\mathrm{eff}} N h_{u} \ell_{L}, \quad$ with $\quad f^{\mathrm{eff}}=f \cos \theta_{N}+\tilde{f} \sin \theta_{N} \frac{\mu^{\prime 2}}{m_{H}^{2}-m_{h}^{2}}$

by considering the large mixing angle $\theta_{N}$ between the neutrinos and also the small mixing between $H_{u}$ and $h_{u}$.

\section{WITH ONE PHASE}

The process (1) can include the phase $\delta_{\mathrm{X}}$ by the interference terms with the diagram with an intermediate $W$ boson. To relate the leptogenesis phase $\delta_{\mathrm{L}}$ to the SM phase(s), one needs a families-unified GUT toward a calculable theory of the physically measurable phases. In the anti-SU(7) [21], indeed, $\delta_{\mathrm{PMNS}}$ and $\delta_{\mathrm{CKM}}$ are shown to be related [11]. In this paper, we attempt to relate the phases in leptogenesis and $\delta_{\text {PMNS }}$ [22]. In other words, we attempt to express the lepton asymmetry $\epsilon_{\mathrm{L}}$ in terms of $\delta_{\text {PMNS }}$. For this, the $W$-boson loop must dominate over the other oneloop corrections.

To obtain a calculable theory for phases, we introduce a single Froggatt-Nielsen(FN) field [23] $X$ developing a complex VEV, $\langle X\rangle=x e^{i \delta_{\mathrm{X}}}[24,25]$. The Yukawa coupling matrix of the doublet $h_{u}$ include powers of $X$ such that some symmetry behind the Yukawa couplings is satisfied. The Yukawa couplings of the three RH neutrinos obtain then a complex phase from different powers of the Froggatt-Nielsen field $X$ depending on the generation. To simplify the discussion, let us assume that the heavy Majorana neutrinos have a mass hierarchy and let the lightest heavy Majorana neutrino $N$ dominate in the leptogenesis calculation.

For the tree $\Delta L \neq 0$ decay mode corresponding to Fig. 1(a), we show the relevant Feynman diagrams interfering in the $N \rightarrow \ell_{j}+h_{u}$ decay in Figs. 1(a) and 1(b) giving rise to a new contribution to leptogenesis. In Figs. 1(a), 1(c), and 1(d), we also show the relevant diagrams for $N \rightarrow \ell_{i}+h_{u}$ decay in the classical leptogenesis scenario, discussed in [26-28]. In the basis where the $N \mathrm{~s}$ and the charged leptons masses are diagonal, possible phases appear at the vertices with the red bullets in Fig. 1. In models where a single complex VEV appears in all the Yukawa couplings, even if with different powers, the classical leptogenesis diagrams given in Figs. 1(c) and 1(d) do not contribute to the $C P$ asymmetry because the overall

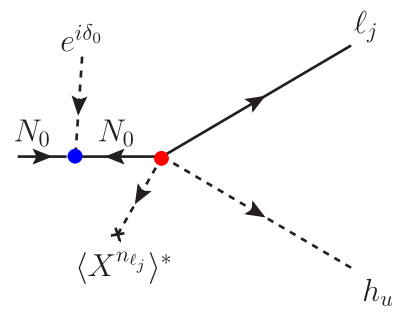

(a)

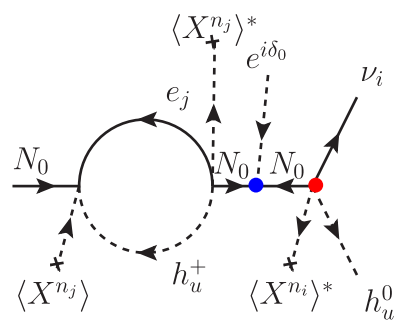

(c)

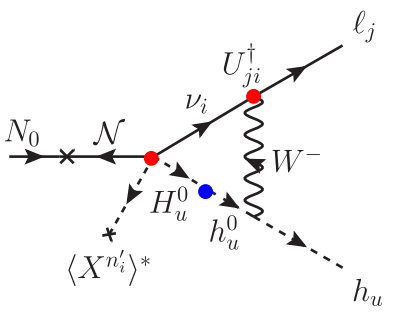

(b)

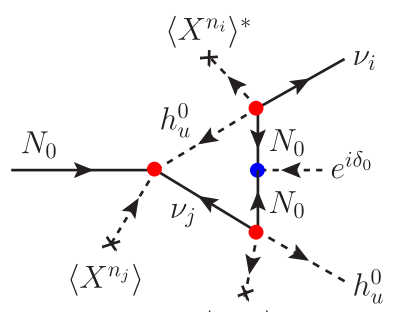

(d)
FIG. 1. The Feynman diagrams interfering in the $N$ decay: (a) the lowest-order diagram, (b) the $W$ exchange diagram, (c) the wave function renormalization diagram, and (d) the heavy neutral lepton exchange diagram. There exist similar $\mathcal{N}$-decay diagrams. In all figures, the final leptons can be both charged leptons and neutrinos. The lepton number violations are inserted with blue bullets, and phases are inserted at red bullets. (a) and (b) interfere. (c) and (d) give a vanishing contribution in $N_{0}$ domination with one complex VEV.

phases cancel out with that of Fig. 1(a). Indeed, we see that in the diagrams the directions of $\left\langle X^{n_{j}}\right\rangle$ are opposite, indicating that the phases are equal and opposite. We are then left to compute the contribution from the diagram with an intermediate $W$. As we will see explicitly below, such contribution vanishes in the presence of a single Yukawa coupling but gives a nonvanishing contribution in our model.

Let us calculate the effect of the vertex correction via the $W$ boson of Figs. 1(b) in the simple mass-insertion formalism. A new conclusion will be drawn from this calculation. With this setup, it is a standard procedure to calculate the asymmetry $\epsilon_{\mathrm{L}}$; i.e., the difference of $N$ decays to the $\ell$ and $\bar{\ell}$,

$$
\epsilon_{\mathrm{L}}^{N}(W)=\frac{\Gamma_{N \rightarrow \ell}-\Gamma_{N \rightarrow \bar{\ell}}}{\Gamma_{N \rightarrow \ell}+\Gamma_{N \rightarrow \bar{\ell}}}
$$

where $\ell(\bar{\ell})$ is a (anti)lepton. We have the following interference term from Figs. 1(a) and 1(b),

$$
\begin{aligned}
\int \frac{d^{4} k}{(2 \pi)^{4}} \mathcal{M}_{(\mathrm{b})} \mathcal{M}_{(\mathrm{a})}^{\dagger}= & i \frac{f_{j} U_{j i}^{\dagger} \tilde{f}_{i}^{*} g_{2}^{2} \mu^{\prime 2}}{2 \sqrt{2}\left(m_{H^{0}}^{2}-m_{h^{0}}^{2}\right)} \\
& \times \mathcal{I}\left(P, p_{\ell}, m_{W}^{2}, m_{h}^{2}, m_{\nu_{i}}^{2}\right),
\end{aligned}
$$

where $\mathcal{I}$ denotes the loop integral depending on the internal masses and the external momenta. 
Here we denote with $\left\{P, m_{0}\right\}$ the four-momentum and mass of $N$, with $\left\{p_{l}, m_{l}\right\}$ the four-momentum and mass of the final state lepton $l_{j}$, while $m_{\nu_{i}}, m_{h^{0(+)}}, m_{H^{0}}$ and $m_{W}$ are the masses of the states $\nu_{i}, h_{u}^{0(+)}, H_{u}^{0}$ and the mass of the $W$ boson, respectively. The $Z$ and photon couplings are flavor diagonal and do not contribute. Note that the factor " $\mu^{\prime 2} /\left(m_{H^{0}}^{2}-m_{h^{0}}^{2}\right)$ " in Eq. (7) is the mixing angle between $h_{u}^{0}$ and $H_{u}^{0}$ in the mass eigenbasis when $\mu^{\prime 2} \ll m_{H^{0}}^{2}, m_{h^{0}}^{2}$. Through such Higgs flavor change at the blue bullet in Fig. 1(b), the lepton number is violated. As in the classical case, the loop integral is UV divergent, but its imaginary part is finite and quite simple in the limit of vanishing mass for the leptons and $m_{W} \ll m_{0}$. Indeed, we obtain
$\operatorname{Im}\left[\mathcal{I}\left(m_{0}^{2}, m_{h^{+}}^{2}, m_{W}^{2}, m_{h^{0}}^{2}, 0\right)\right] \simeq \frac{m_{0}^{2}-m_{h^{0}}^{2}}{8 \pi}\left[1-\ln \left(1+\frac{m_{0}^{2}}{m_{W}^{2}}\right)\right]$,

where we have used $2 P \cdot p_{\ell}=m_{0}^{2}-m_{h^{+}}^{2}$ as set by the kinematical constraints. This expression is IR divergent for vanishing $m_{W}$, but in that limit the PMNS matrix $U_{i j}$ becomes trivial and the $C P$ violation vanishes automatically. Indeed, considering also the neutrino final states in Figs. 1(a) and 1(b), in which case the PMNS matrix is $U$ instead of $U^{\dagger}$, and the analogous diagram with the $W$ loop attached to the tree diagram in Fig. 1(a), we obtain

$$
\epsilon_{L}^{N_{0}}(W)=\frac{\alpha_{\mathrm{em}}}{2 \sqrt{2} \sin ^{2} \theta_{W}} \frac{1}{\sum_{i}\left|f_{i}^{\mathrm{eff}}\right|^{2}} \operatorname{Im}\left[\sum_{i, j}\left[f_{j}^{\mathrm{eff}} U_{j i}^{\dagger}\left(f_{i}^{\mathrm{eff}}\right)^{*}+f_{j}^{\mathrm{eff}} U_{j i}\left(f_{i}^{\mathrm{eff}}\right)^{*}\right]\right] \times\left[1+\ln \left(\frac{m_{W}^{2}}{m_{0}^{2}}\right)\right]
$$

The asymmetry (9) in the limit of unbroken SU(2), but $m_{h^{0}, H^{0}}=m_{h^{+}, H^{+}}$, is given by the simple matrix multiplication $f_{1}^{\dagger}\left(U+U^{\dagger}\right) f_{2}$ where $f_{1,2}$ are column vectors. The imaginary part has the form $\frac{1}{2}\left(f_{1}^{\dagger}\left(U+U^{\dagger}\right) f_{2}-f_{2}^{\dagger}\left(U+U^{\dagger}\right) f_{1}\right)$ which is zero if $f_{1}=f_{2}$ or $\left(U+U^{\dagger}\right)$ is diagonal. This is consistent with the fact that in these diagrams the lepton violation is on the left side of the cut as discussed in [29]. Nevertheless, in our case, thanks to SU(2) breaking, the masses of the particles in the loop are different, so the loop factors are not exactly equal and an imaginary part is present. Indeed, expanding, for example, in the Higgs mass difference $m_{h^{0 /+}}=m_{h}^{2} \mp \frac{1}{2} \Delta m_{h}^{2}$, we obtain instead

$$
\epsilon_{L}^{N_{0}}(W)=\frac{\alpha_{\mathrm{em}}}{2 \sqrt{2} \sin ^{2} \theta_{W}} \frac{\Delta m_{h}^{2}}{m_{0}^{2}} \frac{1}{\sum_{i}\left|f_{i}^{\mathrm{eff}}\right|^{2}} \operatorname{Im}\left[\sum_{i, j}\left[f_{j}^{\mathrm{eff}} U_{j i}^{\dagger}\left(f_{i}^{\mathrm{eff}}\right)^{*}-f_{j}^{\mathrm{eff}} U_{j i}\left(f_{i}^{\mathrm{eff}}\right)^{*}\right]\right] \times\left[1+\ln \left(\frac{m_{W}^{2}}{m_{0}^{2}}\right)\right] .
$$

If the $\mathrm{SU}(2) \times U(1)_{Y}$ is broken, by some choice of $\mathrm{BEH}$ boson couplings $\grave{a}$ la Ref. [14], the mass splitting is $\Delta m_{h}^{2} \propto v^{2}(T)$, and a substantial $C P$ asymmetry is present at $T \leq m_{0}$. Moreover, it is then possible to relate the lepton asymmetry directly to $\delta_{\text {PMNS }}$.

For concreteness, we use the PMNS matrix $U_{i j}$, in the vertex diagram of Fig. 1(b), presented in $[11,30]$ together with Majorana phases $\delta_{a, b, c}$,

$$
U=\left(\begin{array}{ccc}
c_{1} & s_{1} c_{3} & s_{1} s_{3} \\
-c_{2} s_{1} & e^{-i \delta_{\mathrm{PMNS}}} s_{2} s_{3}+c_{1} c_{2} c_{3} & -e^{-i \delta_{\mathrm{PMNS}}} s_{2} c_{3}+c_{1} c_{2} s_{3} \\
-e^{i \delta_{\mathrm{PMNS}} s_{1} s_{2}} & -c_{2} s_{3}+c_{1} s_{2} c_{3} e^{i \delta_{\mathrm{PMNS}}} & c_{2} c_{3}+c_{1} s_{2} s_{3} e^{i \delta_{\mathrm{PMNS}}}
\end{array}\right)_{\mathrm{KS}}\left(\begin{array}{ccc}
e^{i \delta_{a}} & 0 & 0 \\
0 & e^{i \delta_{b}} & 0 \\
0 & 0 & e^{i \delta_{c}}
\end{array}\right)_{\mathrm{Maj}},
$$

where only two phases out of three phases $e^{i \delta_{a, b, c}}$ are independent. Out of three $e^{i \delta_{a, b, c}}$, we choose one freely to match the physics of the problem. As mentioned before, Figs. 1(c) and 1(d) do not have the interference term with (a). So, we choose the Majorana phase of the dominant SM lepton $e^{i \delta_{0}}$ such that it does not have the phase dependence on $e^{i \delta_{0}}$ in the interference with Fig. 1(a). If we assume that the third generation Yukawa couplings dominate, $f_{i}^{\text {eff }}=f_{3}^{\text {eff }} \delta_{i 3}$, we then obtain the expression

$$
\begin{aligned}
\epsilon_{L}^{N_{0}}(W)= & -\frac{\alpha_{\mathrm{em}}}{\sqrt{2} \sin ^{2} \theta_{W}} \frac{\Delta m_{h}^{2}}{m_{0}^{2}} \operatorname{Im}\left[c_{2} c_{3} e^{i \delta_{c}}+c_{1} s_{2} s_{3} e^{i\left(\delta_{\mathrm{PMNS}}+\delta_{c}\right)}\right] \\
& \times\left[1+\ln \left(\frac{m_{W}^{2}}{m_{0}^{2}}\right)\right] .
\end{aligned}
$$

Here we see that the $C P$ asymmetry is directly related to the PNMS phases. The first factor of Eq. (10) is about $10^{-3}$, since the asymmetry is enhanced by the smallness of the $W$ mass. This we will see is an advantage and not a problem since sphaleron transitions are suppressed during the EW symmetry breaking epoch and we can realize baryogenesis even if only a small fraction of the lepton number is converted into the baryon number.

\section{RELATION OF THE PHASES}

Now we can relate the phases in our plan of spontaneous $C P$ violation [31] with one complex VEV, i.e., the phase of $\langle X\rangle$. Following the argument of Ref. [11], we can conclude 
that there will be no observable lepton asymmetry if $\delta_{\mathrm{X}}=0$. Therefore, all the interference terms in Eq. (9) must have factors of the form $\sin \left(N_{i j} \delta_{\mathrm{X}}\right)$ where $N_{i j}$ is an integer. For example, consider the imaginary part of a specific term in Eq. (9) before taking the sum with $i$ and $j$. From the product of Figs. 1(b) and the complex conjugation of 1(a), we read one convenient term, i.e., for $i=3$ and $j=1$, which has the overall phase $e^{i\left[\delta_{\mathrm{PMNS}}+\delta_{a}-n_{1} \delta_{\mathrm{X}}+\delta_{0}\right]+i\left[n_{3} \delta_{\mathrm{X}}-\delta_{0}\right]}$ where $\delta_{\mathrm{PMNS}}$ and $\delta_{a}$ are defined in Eq. (11). The Majorana phase $\delta_{0}$ is the phase of the heavy lepton sector, which does not appear in this phase expression with $i=3$ and $j=1$ if the lightest neutral heavy lepton dominates in the lepton asymmetry. The imaginary part of this term is

$$
\sin \left[\delta_{\mathrm{PMNS}}+\delta_{a}-\left(n_{1}-n_{3}\right) \delta_{\mathrm{X}}\right]
$$

In Ref. [11], we argued that the observable phase $\delta_{\mathrm{PMNS}}$ in low-energy experiments must be integer multiples of $\delta_{\mathrm{X}}$ since there will be no electroweak scale $C P$ violation effects if $\delta_{\mathrm{X}}=0$ and $\pi$. Along this line, we argue that $\delta_{\mathrm{PMNS}}=n_{P} \delta_{\mathrm{X}}$ and $\delta_{a}=n_{a} \delta_{\mathrm{X}}$, which are sufficient for the physical requirement. In this case, Eq. (13) becomes $\sin \left[\left(n_{P}+n_{a}-n_{1}+n_{3}\right) \delta_{\mathrm{X}}\right]$. Now, consider the sum with $i$ and $j$. We observe that each term has the form of $A e^{i\left( \pm n_{P} \delta_{X}+\delta^{\prime}\right)}+B e^{i \delta^{\prime}}$ where $A$ and $B$ are real numbers formed with real angles and $\delta^{\prime}=n^{\prime} \delta_{\mathrm{X}}=n_{a} \delta_{\mathrm{X}}, n_{b} \delta_{\mathrm{X}}$, or $n_{c} \delta_{\mathrm{X}}$, viz. Eq. (11). It is of the form

$$
\begin{aligned}
& \left\{A \cos \left[\left( \pm n_{P}+n^{\prime}\right) \delta_{\mathrm{X}}\right]+B \cos \left[n^{\prime} \delta_{\mathrm{X}}\right]\right\}+i\left\{A \sin \left[\left( \pm n_{P}+n^{\prime}\right) \delta_{\mathrm{X}}\right]+B \sin \left[n^{\prime} \delta_{\mathrm{X}}\right]\right\} \\
& \quad=\sqrt{\left\{A \cos \left[\left( \pm n_{P}+n^{\prime}\right) \delta_{\mathrm{X}}\right]+B \cos \left[n^{\prime} \delta_{\mathrm{X}}\right]\right\}^{2}+\left\{A \sin \left[\left( \pm n_{P}+n^{\prime}\right) \delta_{\mathrm{X}}\right]+B \sin \left[n^{\prime} \delta_{\mathrm{X}}\right]\right\}^{2}} e^{i \delta_{i j}} \equiv a_{i j} e^{i \delta_{i j}},
\end{aligned}
$$

which has the phase $\delta_{i j}=\arctan \left(\left\{A \sin \left[\left( \pm n_{P}+n^{\prime}\right) \delta_{\mathrm{X}}\right]+\right.\right.$ $\left.\left.B \sin \left[n^{\prime} \delta_{\mathrm{X}}\right]\right\} /\left\{A \cos \left[\left( \pm n_{P}+n^{\prime}\right) \delta_{\mathrm{X}}\right]+B \cos \left[n^{\prime} \delta_{\mathrm{X}}\right]\right\}\right)$. Thus, every term has the vanishing phase if $\delta_{\mathrm{X}}=0$ and $\pi$, and the sum in Eq. (13) gives 0 if $\delta_{\mathrm{X}}=0$ and $\pi$. Even at this stage, we have obtained an important conclusion: the phases in the heavy lepton sector do not appear. For further relations, we must use a specific model relating $n_{P}, n^{\prime}, n_{i}$, and $n_{j}$, as we used the flipped-SU(5) model in relating $\delta_{\mathrm{PMNS}}$ and $\delta_{\mathrm{CKM}}[11]$. Thus, the asymmetry takes the form

$$
\epsilon_{\mathrm{L}}^{N_{0}}(W) \approx \frac{\alpha_{\mathrm{em}}}{2 \sqrt{2} \sin ^{2} \theta_{W}} \frac{\Delta m_{h}^{2}}{m_{0}^{2}} \sum_{i, j} \mathcal{A}_{i j} \sin \left[\left( \pm n_{P}+n^{\prime}-n_{i}+n_{j}\right) \delta_{\mathrm{X}}\right]
$$

where $\mathcal{A}_{i j}$ are $a_{i j}$ times appropriate ratio of Yukawa couplings. Note that there are only two independent $n^{\prime}$ as commented before, below Eq. (11).

\section{SPHALERON PROCESSES DURING THE EW BROKEN PHASE}

Contrary to simple approximations, sphaleron transitions [4] are suppressed, but not vanishing when the electroweak symmetry is broken. For a relatively large range of Higgs VEVs, as long as $v \leq T$, one can obtain at least a partial conversion of $L$ into $B$. Indeed, the sphaleron rate in the equilibrium broken phase is given by $[32,33]$

$$
\Gamma_{\mathrm{sph}}^{\text {broken }}=\kappa \alpha_{W}^{4} T^{4}\left(\frac{4 \pi v}{g_{W} T}\right)^{7} e^{-\frac{E_{\mathrm{sph}}}{T}},
$$

where $\kappa$ is a constant, $g_{W}, \alpha_{W}$ are the electroweak coupling and coupling strength and $E_{\mathrm{sph}}$ the energy of the sphaleron energy barrier, proportional to the Higgs VEV, $E_{\mathrm{sph}}=1.524 \pi v / g_{W}$. So, as found in [33], in the SM with a Higgs mass of $125 \mathrm{GeV}$, the sphaleron processes remain in thermal equilibrium until one reaches temperatures of the order $T_{*}=(131.7 \pm 2.3) \mathrm{GeV}$, where $\frac{v}{T}>1$. In case the Higgs VEVs remain nonvanishing, as we advocate here, such VEVs are proportional to the temperature in the high $T$ regime, $v(T)=\sqrt{v(0)^{2}+k^{2} T^{2}}$, as discussed in [14]. Therefore, for $k \sim 1$ the sphaleron processes may enter equilibrium for low temperatures above the electroweak scale $T \geq v(0)$ as long as

$$
\frac{\Gamma_{\mathrm{sph}}^{\mathrm{broken}}}{T^{3} H(T)}=\kappa \alpha_{W}^{4}\left(\frac{4 \pi k}{g_{W}}\right)^{7} e^{-1.52 k \frac{4 \pi}{g_{W}}} \sqrt{\frac{90}{\pi^{2} g_{*}}} \frac{M_{P}}{T} \geq 1 .
$$

In this case, the sphaleron processes are active only for a very short range of temperatures, nevertheless a partial conversion of lepton number into baryon number may still be possible, giving rise to the observed baryon asymmetry if the lepton number is sufficiently large. In that case, the solution of the system of Boltzmann equations for the baryon and lepton numbers is needed to obtain the final baryon asymmetry, which we leave to be studied in detail in a future publication. 
In the most favorable case of efficient sphaleron transitions at $T \geq v(0)$, able to fully equilibrate the baryon and lepton numbers, one obtains simply [34]

$$
\eta_{B} \sim 10^{-2} \kappa \epsilon_{L},
$$

so we need $\epsilon_{L} \simeq 6 \times 10^{-6}$ to obtain the observed baryon asymmetry, in the strong wash-out regime $\kappa \sim 10^{-2}$ for the $B-L$ number production at $T \sim 0.1 m_{0}$. Then, in the simplified case of hierarchical Yukawa given in Eq. (12), we have for $\Delta m_{h} \propto v(t) \sim T$, the constraint

$c_{2} c_{3} \sin \delta_{c}+c_{1} s_{2} s_{3} \sin \left(\delta_{c}+\delta_{\mathrm{PMNS}}\right) \simeq 2.4 \times 10^{-2}$,

so phases of order $10^{-2}$ are sufficient to generate the baryon asymmetry. Indeed, for $\delta_{c}=0$, we obtain a lower bound on the PMSN phase as $\sin \delta_{\text {PMNS }} \geq 0.06$.

In case a larger $C P$ violation is present or in the weak wash-out regime $\kappa \sim 1$, the generated $B-L$ yield is larger, and a suppression of the final baryon number can be achieved by partial conversion through out-of-equilibrium sphaleron transitions. Note that, in the general case, there is also a dependence on the effective Yukawas $f_{i}^{\text {eff }}$, via the coefficients $a_{i j}$ in Eq. (15), and on the Majorana phases.

\section{CONCLUSION}

By introducing only one $C P$ phase by a complex VEV of a SM singlet field $X$ and assuming leptogenesis via the lightest Majorana neutrino out of equilibrium decay during a phase where the electroweak symmetry is broken, we have that the dominant contribution to the $C P$ asymmetry in the early Universe arises from a $W$-boson loop, directly containing the PMNS phase $\delta_{\text {PMNS }}$. We have shown that such $C P$ violation is able to account for the present baryon asymmetry without any stretch of the parameters. Indeed, in the most favorable case, small PMNS phases of order $10^{-1}-10^{-2}$ are sufficient to give the baryon number, but in the case of nonperfect equilibration for the sphaleron transitions, larger phases $\mathcal{O}(1)$ may be needed in order to overproduce the lepton number. In both cases, we predict a nonvanishing PMNS phase, possibly measured in future neutrino experiments.

In this scenario, we are able to have a novel mechanism to relate high- and low-energy $C P$ violation, in particular in the case when a single $C P$ phase is introduced by spontaneous mechanism at a high-energy scale along the Froggatt-Nielsen method. Even if more phases and a nonvanishing contribution from the classical loop with the heavier RH neutrinos states are present, the PMNS phase could still represent the dominant part and allow for a direct correlation of the baryon asymmetry to neutrino observables.

\section{ACKNOWLEDGMENTS}

We thank S. M. Barr for useful comments. L. C. acknowledges partial financial support by the European Union's Horizon 2020 research and innovation program under the Marie Sklodowska-Curie Grant Agreements No. 690575 and No. 674896. J.E. K. is supported in part by the National Research Foundation (NRF) grant funded by the Korean Government (MEST) (NRF-2015R1D1A1A01058449) and the IBS (IBS-R017-D1-2016-a00), B. K. is supported in part by the NRF-2013R1A1A2006904 and Korea Institute for Advanced Study grant funded by the Korean government, and S. N. is supported in part by NRF-2013R1A1A2004538.
[1] S. Davidson and A. Ibarra, Leptogenesis and low-energy phases, Nucl. Phys. B648, 345 (2002); E. W. Kolb and M. S. Turner, The Early Universe, Frontiers in Physics, Vol. 69 (Addison-Wesley, Reading, MA, 1990), p. 160.

[2] A. D. Sakharov, Violation of $C P$ invariance, $\mathrm{C}$ asymmetry, and baryon asymmetry of the universe, Pis'ma Zh. Eksp. Teor. Fiz. 5, 32 (1967) [JETP Lett. 5, 24 (1967)]; Usp. Fiz. Nauk 161, 61 (1991) [Sov. Phys. Usp. 34, 392 (1991)].

[3] M. Yoshimura, Unified Gauge Theories and the Baryon Number of the Universe, Phys. Rev. Lett. 41, 281 (1978).

[4] V. A. Kuzmin, V. A. Rubakov, and M. E. Shaposhnikov, On the anomalous electroweak baryon number nonconservation in the early universe, Phys. Lett. 155B, 36 (1985).

[5] I. Affleck and M. Dine, A new mechanism for baryogenesis, Nucl. Phys. B 249, 361 (1985).

[6] M. Fukugita and M. Yanagida, Baryogenesis without grand unification, Phys. Lett. B 174, 45 (1986).
[7] H. D. Kim, J. E. Kim, and T. Morozumi, A new mechanism for baryogenesis living through electroweak era, Phys. Lett. B 616, 108 (2005).

[8] N. Cabibbo, Unitary Symmetry and Leptonic Decays, Phys. Rev. Lett. 10, 531 (1963).

[9] M. Kobayashi and T. Maskawa, $C P$ violation in the renormalizable theory of weak interaction, Prog. Theor. Phys. 49, 652 (1973).

[10] B. Pontecorvo, Mesonium and anti-mesonium, Zh. Eksp. Teor. Fiz. 33, 549 (1957) [Phys. JETP 6, 429 (1957)]; Z. Maki, M. Nakagawa, and S. Sakata, Remarks on the unified model of elementary particles, Prog. Theor. Phys. 28, 870 (1962).

[11] J. E. Kim and S. Nam, Unifying $C P$ violations of quark and lepton sectors, Eur. Phys. J. C75, 619 (2015).

[12] S. M. Barr, G. Segré, and H. A. Weldon, The magnitude of the cosmological baryon asymmetry, Phys. Rev. D 20, 2494 (1979). 
[13] M. B. Gavela, P. Hernandez, J. Orlo, O. Pene, and C. Quimbay, Standard Model $C P$-violation and baryon asymmetry Part II: Finite temperature, Nucl. Phys. B430, 382 (1994).

[14] R. N. Mohapatra and G. Senjanovic, Broken symmetries at high temperature, Phys. Rev. D 20, 3390 (1979).

[15] L. E. Ibanez and G. Ross, $\mathrm{SU}(2)_{L} \times U(1)$ symmetry breaking as a radiative effect of supersymmetry breaking in GUTs, Phys. Lett. 110B, 215 (1982); J. R. Ellis, J. S. Hagelin, D. V. Nanopoulos, and K. Tamvakis, Weak symmetry breaking by radiative corrections in broken supergravity, Phys. Lett. 125B, 275 (1983).

[16] D. V. Nanopoulos and S. Weinberg, Mechanism for cosmological baryon production, Phys. Rev. D 20, 2484 (1979).

[17] A. Bhattacharya, R. Gandhi, and S. Mukhopadhyay, Revisiting the implications of $C P T$ and unitarity for baryogenesis and leptogenesis, Phys. Rev. D 89, 116014 (2014); For a recent discussion, see, F. Rompineve, Weak scale baryogenesis in a supersymmetric scenario with R-parity violation, J. High Energy Phys. 08 (2014) 014.

[18] G. C. Branco, R. Gonzalez Felipe, F. R. Joaquim, I. Masina, M. N. Rebelo, and C.A. Savoy, Minimal scenarios for leptogenesis and $C P$ violation, Phys. Rev. D 67, 073025 (2003).

[19] A. Abada, S. Davidson, A. Ibarra, F.-X. Josse-Michaux, M. Losada, and A. Riotto, Flavour matters in leptogenesis, J. High Energy Phys. 09 (2006) 010.

[20] S. Pascoli, S. T. Petcov, and A. Riotto, Leptogenesis and low energy $C P$ violation in neutrino physics, Nucl. Phys. 774, 1 (2007).

[21] J. E. Kim, Towards unity of families: anti-SU(7) from $\mathbf{Z}_{12-I}$ orbifold compactification, J. High Energy Phys. 06 (2015) 114 [arXiv:1503.03104].

[22] For an earlier try, see, G. C. Branco, P. A. Parada, and M. N. Rebelo, A common origin for all $C P$ violations, arXiv:hep-ph/0307119.
[23] C. D. Froggatt and H. B. Nielsen, Hierarchy of quark masses, Cabibbo angles and $C P$ violation, Nucl. Phys. 147, 277 (1979).

[24] J. E. Kim, The CKM matrix with maximal $C P$ violation from Z(12) symmetry, Phys. Lett. B 704, 360 (2011).

[25] J. E. Kim, D. Y. Mo, and M.-S. Seo, The CKM matrix from anti-SU(7) unification of GUT families, Phys. Lett. B 749 , 476 (2015).

[26] L. Covi, E. Roulet, and F. Vissani, $C P$ violating decays in leptogenesis scenarios, Phys. Lett. B 384, 169 (1996).

[27] M. Flanz, E. A. Paschos, and U. Sarkar, Baryogenesis from a lepton asymmetric Universe, Phys. Lett. B 345, 248 (1995); 382, 447(E) (1996).

[28] W. Buchmüller and M. Plümacher, $C P$ asymmetry in Majorana neutrino decays, Phys. Lett. B 431, 354 (1998).

[29] R. Adhikari and R. Rangarajan, Baryon number violation in particle decays, Phys. Rev. D 65, 083504 (2002).

[30] J. E. Kim and M-S. Seo, Parametrization of the CKM matrix, Phys. Rev. D 84, 037303 (2011); J. E. Kim, D. Y. Mo, and S. Nam, Final state interaction phases obtained by data from $C P$ asymmetries, J. Korean Phys. Soc. 66, 894 (2015)

[31] T. D. Lee, A theory of spontaneous T violation, Phys. Rev. D 8, 1226 (1973).

[32] Y. Burnier, M. Laine, and M. Shaposhnikov, Baryon and lepton number violation rates across the electroweak crossover, J. Cosmol. Astropart. Phys. 02 (2006) 007.

[33] M. D’Onofrio, K. Rummukainen, and A. Tranberg, Sphaleron Rate in the Minimal Standard Model, Phys. Rev. Lett. 113, 141602 (2014); The sphaleron rate through the electroweak cross-over, J. High Energy Phys. 08 (2012) 123.

[34] W. Buchmuller, P. Di Bari, and M. Plumacher, Leptogenesis for pedestrians, Ann. Phys. (Amsterdam) 315, 305 (2005). 